Cahiers de philosophie de l'université de

Levinas : au-delà du visible

\title{
Préface - Les inédits de Levinas : la genèse d'une pensée
}

Jean-Luc Marion

\section{(2) OpenEdition}

\section{Journals}

Édition électronique

URL : https://journals.openedition.org/cpuc/766

DOI : 10.4000/cpuc.766

ISSN : 2677-6529

Éditeur

Presses universitaires de Caen

\section{Édition imprimée}

Date de publication : 31 juillet 2012

Pagination : 9-12

ISBN : 978-2-84133-410-0

ISSN : $1282-6545$

\section{Référence électronique}

Jean-Luc Marion, «Préface - Les inédits de Levinas : la genèse d'une pensée », Cahiers de philosophie de l'université de Caen [En ligne], 49 | 2012, mis en ligne le 07 juin 2018, consulté le 02 février 2023. URL : http://journals.openedition.org/cpuc/766 ; DOI : https://doi.org/10.4000/cpuc.766

\section{(c) (†) \&)}

Creative Commons - Attribution - Pas d'Utilisation Commerciale 4.0 International - CC BY-NC 4.0 https://creativecommons.org/licenses/by-nc/4.0/ 


\section{Préface}

\section{Les inédits de Levinas: la genèse d'une pensée}

$\mathrm{L}$

ES TEXTES PRÉSENTS dans cet ouvrage mettent en lumière l'importance des deux volumes d'inédits publiés récemment: Carnets de captivité et autres inédits et Parole et Silence et autres conférences inédites au Collège philosophique (Paris, Grasset - IMEC, 2009 et 2011). La philosophie est par essence système et si tous les écrits d'un auteur n'ont pas à entrer dans le système, ces deux volumes d'inédits relèvent directement du système de cette pensée en genèse qui aboutira à Totalité et Infini (1961). Car c'est bien, pour l'essentiel, la genèse de cet ouvrage, premier exposé fondamental de la pensée lévinassienne, que nous permettent de suivre ces inédits. Cependant, dans la mesure où le difficile travail d'interprétation proposé dans ce numéro des Cahiers de philosophie de l'Université de Caen s'intéresse à la genèse du système, il fait apparaître également les possibilités que ce dernier ne retiendra pas, qui finiront par se refermer, comme ici la réflexion sur la métaphore, qui tente, juste après Totalité et Infini, d'ouvrir un chemin vers la pensée de l'autrement qu'être, pensée qui ne connaîtra sa véritable formulation que plus de dix années après Totalité et Infini. En proposant de lire l'œuvre de Levinas à partir de ses commencements plutôt que par sa conclusion, il permet ainsi de ne pas lui imposer une téléologie trop rigide, et de se laisser surprendre par d'inattendus chemins (l'exploration de la voie romanesque, ou encore la lecture de penseurs chrétiens).

Il demeure que ces textes inédits ne sont pas les premiers de Levinas et qu'il est impossible de les élucider sans les confronter à De l'évasion, qui marque véritablement la naissance de la philosophie de Levinas par une rupture radicale qui est également une percée. En mettant en évidence en 1935 que l'être se caractérise par sa "suffisance» et soumet l'existant 
à un «asservissement», Levinas dévoile le besoin de sortir de soi: «Dans l'identité du moi, l'identité de l'être révèle sa nature d'enchaînement car elle apparaît sous forme de souffrance et elle invite à l'évasion ${ }^{1}$. Or la radicalité du geste tient au fait qu'il ne s'agit pas de s'évader d'une modalité de l'être comme l'être fini, mais bien de l'être en tant qu'être, qui est en lui-même imparfait. Levinas peut déjà écrire: «L'être du moi que la guerre et l'après-guerre nous ont permis de connaître ne nous laisse plus aucun jeu. Le besoin d'en avoir raison ne peut-être qu'un besoin d'évasion ${ }^{2}$. En effet Levinas, qui voyait depuis la Première Guerre mondiale le développement du nihilisme et pressentait la catastrophe à venir, ne cherchait pas dans la purification toujours plus poussée de la volonté une issue à la crise de l'Europe, puisqu'elle maintenait l'enchaînement dans l'être, source du nihilisme. Seul l'autrement qu'être, dont Levinas donnera l'analyse systématique en 1974, sera un affranchissement de l'être.

On comprend alors que les Carnets de captivité ne se contentent pas de décrire le difficile quotidien des prisonniers. Levinas, en vrai penseur, réfléchit sur le sens de la captivité, sans prendre pour autant l'attitude du spectateur extérieur. En philosophe, il s'interroge sur la captivité du sens, c'est-à-dire sur le statut de l'il $y a$, de l'hypostase et de l'être, pour décrire ce dont il s'agit de s'évader. Qu'est-ce que Levinas veut libérer? Il veut libérer le sens de son enfermement dans l'être, et donc dans le langage de la métaphysique, pour donner à redécouvrir le «pour autrui» du sens, le langage comme invocation, comme un enseignement qui n'est pas maïeutique.

Les pages qui suivent rassemblent en effet des efforts remarquables - et remarquablement nouveaux - pour identifier ce d'où part Levinas et comment il s'en départit, reconstituant ainsi une manière de pré-histoire de sa philosophie déjà connue, parfois il est vrai à contresens. Le lecteur bienveillant constatera très vite la profonde cohérence de l'ensemble d'études ici rassemblées. On a distribué l'ensemble en quatre parties égales, qui se relaient exactement. L'introduction déjà reconnaitt la parole au son (Michael Levinas), et, par contraste, le dossier sur la soutenance de thèse du 6 juin 1961, qui constitue la première partie, fait voir l'origine de la presque complète mécompréhension par le jury de Totalité et Infini: mécompréhension pour y avoir lu une doctrine philosophique, au lieu d'y entendre la lente construction, en fait procédant plutôt par destruction, d'une parole d'appel et d'écoute. Ici une erreur d'un typographe dans le rapport de Jacques

1. De l'évasion, introduit et annoté par J. Rolland, Montpellier, Fata Morgana, 1982 et Paris, Librairie générale française (Le Livre de poche), 1998, p. 98.

2. Ibid., p. 95 . 
Taminiaux, que relate ce dernier, prend tout son sens involontaire: «D'où la substitution malheureuse du mot déduction au mot réduction [...]. Il faut lire réduction phénoménologique et non pas déduction phénoménologique. À aucun moment la phénoménologie dont se réclamait Levinas n'est une affaire de déduction» (p. 81). En effet, mais jusqu'où va cette réduction et comment procède-t-elle? C'est ce que, dans la deuxième partie, Danielle Cohen-Levinas tente de préciser en pensant le son comme une parole, parce que, plus radicalement encore, la parole sonne, et, sonnant, résonne comme une adresse. Ce qui permet à Dan Arbib, à partir de la conférence "Parole et Silence» (1948), de découvrir que l'événement du son anticipe sur ce que, après un "étouffement» provisoire, le visage accomplira en 1961, l'appel. Il se pourrait alors que, même si cette tentative sera finalement abandonnée (Rodolphe Calin), les analyses de la «hauteur» du langage dans la métaphore (Marc Faessler) visent le même but: inscrire l'extériorité dans le langage, lui-même décrit à partir de la parole (et non de la langue, ni surtout stricto sensu du langage en son acception saussurienne), en sorte de le mettre en œuvre, ou plutôt de se laisser mettre en œuvre par lui comme appel et réponse (Francis Guibal). La troisième partie dégage la tentative répétée d'une sortie comparable vers la transcendance, cette fois à partir de la captivité elle-même. D’abord avec la mise à nu de l'il y $a$, dans la quasiscène primitive des "draperies d'Alençon" arrachées par les dévastés de la débâcle (François-David Sebbah); ensuite par l'épreuve du besoin (la faim) des prisonniers (Zinaida Sokuler); puis par l'incompatibilité entre être et salut (Bernhard Casper) et enfin le "monde cassé » (Emmanuel Housset), qui aboutit à la conclusion globale déjà énoncée par De l'évasion: "Aussi l'évasion est-elle le besoin de sortir de soi-même, c'est-à-dire de briser l'enchaînement le plus radical, le plus irrémissible, le fait que le moi est soi-même» ${ }^{3}$.

La question de l'eros peut alors s'aborder, dans une quatrième partie, comme une dernière tentative d'évasion. D'abord comme retournement, Umkherung plus radicale encore que la réduction en style husserlien, renversement qui dégage un «envers sans endroit» (Carla Canullo). Ensuite comme une jouissance, dont Corine Pelluchon suggère qu'elle conduit et contraint à la justice. Enfin, figure supérieure de cette justice, l'enseignement, qui, selon la démonstration convaincante de Henrik Vase Frandsen, n’appartient pas à la maïeutique, mais relève de la révélation immédiate de la loi, altérité et transcendance pures. Il ne faut certes pas attribuer à un effet de hasard que Gérard Bensussan puisse conclure, citant les Carnets,

3. Ibid., p. 98. 
que l'«évasion dans quelque chose qui n'est pas l'être» a finalement pour nom "amour», anticipant ainsi sur des déclarations plus tardives et d'autant plus décisives que Levinas avait beaucoup résisté à l'emploi du terme ${ }^{4}$.

\author{
Jean-Luc MARION \\ de l'Académie française \\ Professeur émérite à l'université Paris-Sorbonne \\ Professeur à The University of Chicago \\ Professeur à l'Institut catholique de Paris
}

4. Ainsi: «Ce qui est vraiment humain, c'est - ne vous effrayez pas de ce mot - l'amour» (entretien avec Christoph von Wolzogen, trad. fr. A. David, Philosophie, $\mathrm{n}^{\circ}$ 93, printemps 2007, p. 15). Voir Autrement que savoir, G. Petitdemange et J. Rolland (éd.), Paris, Osiris, 1988, p. 75. 